\title{
Association of Aggression With a Novel MicroRNA Binding Site Polymorphism In the Wolframin Gene
}

\begin{tabular}{|r|l|}
\hline Journal: & American Journal of Medical Genetics Part B: Neuropsychiatric Genetics \\
\hline Manuscript ID: & NPG-12-0187.R1 \\
\hline Wiley - Manuscript type: & Research Article \\
\hline Date Submitted by the Author: & n/a \\
\hline Complete List of Authors: & $\begin{array}{l}\text { Kovacs-Nagy, Reka; Semmelweis University, Department of Medical } \\
\text { Chemistry } \\
\text { Elek, Zsuzsanna; Semmelweis University, Department of Medical } \\
\text { Chemistry } \\
\text { Szekely, Anna; Eotvos Lorand University, Institute of Psychology } \\
\text { Nanasi, Tibor; Semmelweis University, Department of Medical Chemistry } \\
\text { Sasvari-Szekely, Maria; Semmelweis University, Department of Medical } \\
\text { Chemistry } \\
\text { Ronai, Zsolt; Semmelweis University, Department of Medical Chemistry }\end{array}$ \\
\hline Keywords: & \begin{tabular}{l} 
aggression, microRNA, WFS1, rs1046322, SNP \\
\hline
\end{tabular} \\
\hline
\end{tabular}

\section{SCHOLARONE ${ }^{m}$}

Manuscripts 
Kovacs-Nagy et al. WFS1 and aggression

\title{
ASSOCIATION OF AGGRESSION WITH A NOVEL MICRORNA BINDING SITE POLYMORPHISM IN THE WOLFRAMIN GENE
}

\author{
Reka Kovacs-Nagy ${ }^{1}$, Zsuzsanna Elek ${ }^{1}$, Anna Szekely ${ }^{2}$, Tibor Nanasi ${ }^{1}$, Maria Sasvari- \\ Szekely ${ }^{1}$, Zsolt Ronai $^{1}$ \\ ${ }^{1}$ Institute of Medical Chemistry, Molecular Biology and Pathobiochemistry, Semmelweis \\ University, Budapest, Hungary \\ ${ }^{2}$ Institute of Psychology, Eötvös Loránd University, Budapest, Hungary \\ Corresponding author: Zsolt Ronai \\ Tuzolto u. 37-47. Budapest, H-1094 Hungary \\ Tel: $+3620-6660135$ \\ Fax: $+3620-6660104$ \\ Email: ronai.zsolt@med.semmelweis-univ.hu
}

Running Head: WFS1 and aggression

Keywords: aggression, microRNA, WFS1, rs 1046322, SNP

John Wiley \& Sons, Inc. 
Kovacs-Nagy et al. WFS1 and aggression

\begin{abstract}
Background

Rare mutations in the WFS1 gene lead to Wolfram syndrome, a severe multisystem disorder with progressive neurodegeneration and diabetes mellitus causing life-threatening complications and premature death. Only a few association studies using small clinical samples tested the possible effects of common WFS1 gene variants on mood disorders and suicide, the non-clinical spectrum has not been studied yet.
\end{abstract}

\title{
Methods
}

Self-report data on Aggression, Impulsiveness, Anxiety and Depression were collected from a large $(\mathrm{N}=801)$ non-psychiatric sample. SNPs were selected to provide an adequate coverage of the entire WFS1 gene, as well as to include putative microRNA binding site polymorphisms. Molecular analysis of the assumed microRNA binding site variant was performed by an in vitro reporter-gene assay of the cloned 3' UTR with coexpression of miR668.

\section{Results}

Among the 17 WFS1 SNPs, only the rs1046322, a putative microRNA (miR-668) binding site polymorphism showed significant association with psychological dimensions after correction for multiple testing: those with the homozygous form of the minor allele reported higher aggression on the Buss-Perry Aggression Questionnaire ( $p=0.0005)$. Functional effect of the same SNP was also demonstrated in a luciferase reporter system: the minor A allele showed lower repression compared to the major $\mathrm{G}$ allele, if co-expressed with miR-668.

\section{Conclusions}

To our knowledge, this is the first report describing a microRNA binding site polymorphism of the WFS1 gene and its association with human aggression based on a large, non-clinical sample. 
Kovacs-Nagy et al. WFS1 and aggression

\section{INTRODUCTION}

Wolfram syndrome (WS) is an autosomal recessive multisystem disorder with progressive neurodegeneration and diabetes mellitus [Rigoli and others 2011], also known as DIDMOAD (diabetes insipidus, diabetes mellitus, optic atrophy and deafness). Although the clinical course of WS is highly variable, the prognosis is poor as patients have an impaired quality of life and usually die in their thirties from central respiratory failure as a result of brainstem atrophy [Barrett and others 1995]. Psychiatric manifestations are common in WS patients, including severe depression, psychosis, as well as impulsive verbal and physical aggression [Swift and others 1990].

The mutated gene, WFS1 was identified in 1998 on chromosome 4p16.1 spanning $33.4 \mathrm{~kb}$ [Inoue and others 1998]; it has eight exons but the first exon is noncoding. The WFS1 gene encodes a transmembrane protein (wolframin) of the endoplasmatic reticulum (ER), predominantly expressed in the brain and in the pancreatic $\beta$ cells, but detected nearly in all tissues. Although the molecular function of wolframin is not fully understood to date, current results suggest that wolframin has a critical role in the regulation of ER stress [Fonseca and others 2005; Fonseca and others 2010]. ER homeostasis is based on a sensitive balance of protein synthesis and protein folding capacity of the ER. Accumulation of unfolded proteins induces ER stress, triggering the unfolded protein response (UPR). At the same time it is of vital importance to avoid UPR hyperactivation, which could trigger apoptotic pathways and lead to cell death. According to recent data, wolframin might participate in down regulation of ER stress and thus, in the reestablishment of ER homeostasis [Oslowski and Urano 2011]. Further findings pointed out another possible role of wolframin in controlling ER calcium homeostasis [Takei and others 2006]. A recent study demonstrated that wolframin is a calcium-calmodulin binding protein, and three mutations found in WS patients completely abolish this function [Yurimoto and others 2009]. Taken together the findings above about the 
Kovacs-Nagy et al. WFS1 and aggression

molecular function of wolframin, this protein is expected to have a vital role in ER homeostasis of excretory cells, such as the insulin producing pancreatic $\beta$ cells or the neurons producing synaptosomes for neurotransmission.

Studies on the WFS1 knockout mouse provided further evidence for the significant role of wolframin in the brain and in the pancreas. WFS1 deficient mice had a dramatic decrease in the mass of the pancreatic islets and a serious impairment of glucose homeostasis [Ishihara and others 2004]. Results from behavioral studies of the WFS1 knockout mouse suggested that it could be used as an animal model of mood disorder [Kato and others 2008], impaired behavioral stress adaptation [Luuk and others 2009] and post-traumatic stress disorder [Kesner and others 2009].

Although more than 200 different mutations causing WS have been identified (for a recent database see: [M. 2010]), phenotype-genotype relationships are still not clear. Similarly, little is known about the clinical and molecular effects of common variants. Several single nucleotide polymorphisms (SNPs) have been shown as possible risk factors for type 2 diabetes mellitus, such as the rs10010131 in intron 4 and rs1046320 in the 3' untranslated region (UTR) [Fawcett and others 2010]. Other studies raised the possibility of association between several WFS1 SNPs and neuropsychiatric disorders, such as mood disorders [Kawamoto and others 2004; Koido and others 2005] and impulsive suicide [Lemonde and others 2003; Must and others 2009]. Although the level of significance did not survive corrections for multiple testing in most studies, the possible link between WFS1 genetic variants and psychiatric disorders is strongly supported by the fact that carriers of various WFS1 mutations are 26-fold more likely to be hospitalized in psychiatric institutions than noncarriers [Swift and Swift 2000].

According to our best knowledge, WFS1 polymorphisms have not been studied yet in relation to either aggression or mood characteristics within the non-clinical spectrum. Here we present 
Kovacs-Nagy et al. WFS1 and aggression

an association study of WFS1 SNPs and four psychological dimensions: Aggression, Impulsiveness, Anxiety, and Depression, using self-report data from a large, non-psychiatric sample. Study of these phenotypes was based on the main psychiatric symptoms of WS patients (depression and aggression), as well as on the results of the few psychogenetic association studies related to mood disorders and impulsive suicide. In addition, we characterized the molecular function of rs1046322 which had a significant effect on the measured phenotypes.

\section{MATERIALS AND METHODS}

\section{Sample}

Caucasian (Hungarian) participants of the study signed written informed consent, provided buccal samples and filled out questionnaires. They were recruited at different higher education sites on a voluntary basis. The study protocol was approved by the Scientific and Research Ethics Committee of the Medical Research Council (ETT TUKEB). Valid DNA and questionnaire data were available from 801 subjects (46.2\% males and 53.8\% females). Mean age was 21.3 years $( \pm 3.3)$ within the age range of $18-35$ years.

\section{Phenotypic Measures}

All subjects completed a panel of self-report measures relevant to the study of Aggression, Impulsiveness, Anxiety and Depression. The Buss-Perry Aggression Questionnaire [Buss and Perry 1992] includes 29 items, rated from 1 (extremely uncharacteristic of me) to 5 (extremely characteristic of me). The aggression construct was measured with items loading on four distinct factors: Verbal Aggression (5 items), Anger (7 items), Hostility (8 items) and Physical Aggression (9 items). The total score for Aggression was the sum of ratings for all the items, thus possible total scores range from 29 to 145 . The $11^{\text {th }}$ version of the Barratt 
Kovacs-Nagy et al. WFS1 and aggression

Impulsiveness Scale was designed to assess general impulsiveness using 30 items scored from 1-4 (BIS-11; [Patton and others 1995]). In the present study the total score for Impulsiveness was used, with values ranging from 30-120. The English version of the questionnaires measuring aggression and impulsiveness were translated using the 'forward-backward' procedure and were pilot tested prior to the present study to enhance translation clarity and applicability. Anxiety and depression was measured by the Hungarian version of the Hospital Anxiety and Depression Scale (HADS; [Muszbek and others 2006]). Items are scored from 03 based on the selected response category, possible total scores for both anxiety ( 7 items) and depression (7 items) range from 0 to 21.

To assess the internal consistency, Cronbach alpha values were calculated for each phenotype construct in the present sample, and were found satisfactory (Aggression Total Score: 0.90; Impulsiveness Total Score: 0.82; Anxiety Scale: 0.77; Depression Scale: 0.74). Internal consistency measures of the four aggression factors were also sufficient (Verbal Aggression: 0.64; Anger: 0.84; Hostility: 0.80; Physical Aggression: 0.84).

\section{Marker Selection}

Common (higher than $5 \%$ minor allele frequency) polymorphic loci were selected from dbSNP database using HapMap information about linkage disequilibrium to provide adequate coverage of the entire WFS1 gene. LD blocks were defined by the "Four Gamete Rule" using Haplowiev 4.2. At least two SNPs have been investigated in each block taking the chromosomal localization also into consideration. Our goal was to generate a balanced "physical coverage" so that no segments longer than $6 \mathrm{~kb}$ remained uncovered. Priority was given to those SNPs which were referred in various association studies in connection with diabetes mellitus and psychiatric disorders. SNPs, theoretically influencing microRNA binding, were searched in the polymiRTS and Patrocles databases. 
Kovacs-Nagy et al. WFS1 and aggression

\section{DNA and genotyping}

Genomic DNA was extracted from buccal swabs by Gentra purification kit (Minneapolis, US) according to manufacturer's instructions and quantified by fluorometry employing an intercalation assay (AccuBlue Broad Range dsDNA Quantitation Kit, Biotium, Hayward, CA). Typical DNA concentration was about $50 \mathrm{ng} / \mu \mathrm{l}$ (range: $15-200 \mathrm{ng} / \mu \mathrm{l}$ ).

Genotyping was performed by an Open Array ${ }^{\circledR}$ real-time PCR platform of Applied Biosystems (Foster City, CA) based on the 5' nuclease assay with pre-designed and validated primers and allele-specific, fluorescent (TaqMan) probes immobilized to a solid surface. Approximately 100 ng (range: 30-150 ng) DNA was applied for each SNP subarray. The master mix, containing each dNTP and the AmpliTaq Gold DNA-polymerase, was supplied by the manufacturer. The thermocycle was carried out according to the manufacturer's instruction. Allele specific FAM and VIC fluorescent intensities were measured after the PCR amplification, genotypes were called using the TaqMan Genotyper v1.2 software. Call rates for SNPs on the OpenArray system was $88.87 \%$ in average (range: 69.37-96.00\%). For quality control, three SNPs (rs9457, rs1046320 and rs1046322) were genotyped additionally on a conventional 96 well plate system with the commercially available TaqMan assay kits on a 7300 Real-Time PCR System (Applied Biosystems, Foster City, CA). Re-genotyping proved the reliability of the high-throughput OpenArray platform, as $99.1 \%$ agreement was observed comparing the two approaches, moreover increased the call rate of genotypes at these 3 loci. The genotype and allele frequencies obtained as a combination of data determined by the two genotyping platforms for the 801 individuals with valid data of questionnaires were used for association analysis between genotypes and phenotypic measures. Genotype frequency data for each SNP are given in Supplementary Table 1, minor allele frequencies (MAF) are shown in Table 1. The measured genotype frequencies 
Kovacs-Nagy et al. WFS1 and aggression

corresponded to the Hardy-Weinberg equilibrium for all SNPs (for $p$ values see: Supplementary Table 1). Call rates for each SNP were calculated as the sum of the genotypes given in Supplementary Table 1 divided by the total number of participants with valid phenotypic data (801).

\section{Luciferase constructs of the WFS1 3' UTR}

The entire 3' UTR region of the human WFS1 gene was amplified by PCR of a sample with known rs1046322 (GG) genotype using the 5' TCG GCG GAG CTC GGA TGG TCC GCC ACG AGG AGC 3' forward and 5' AAA GGA AAG CTT GCG CTG CAG GTT CCA CCA GAG G 3' reverse primers. Bold letters indicate the Sac I and Hind III recognition sites in the forward and reverse primer, respectively. PCR product was purified and cloned between the Sac I and Hind III sites downstream to the firefly luciferase gene in pMIR-Report vector (Ambion) by standard protocols (pMIR-G). Construct with WFS1 3' UTR possessing the rs1046322 A allele (designated pMIR-A) was generated by quick change site directed mutagenesis of the cloned pMIR-G, both constructs were confirmed by direct sequencing. A control pMIR construct (pMIR-c) was also created by cloning a DNA-fragment with identical length, however without the binding site of miR-668 downstream to the luciferase gene.

\section{Cell transfection and luciferase assay}

The Human Embryonic Kidney 293 (HEK293) cell line was cultured in DMEM medium (Invitrogen-Gibco), supplemented with $10 \%$ bovine fetal serum (Lonza) at $37{ }^{\circ} \mathrm{C}$ in an atmosphere of 5\% $\mathrm{CO}_{2}$. HEK293 cells were transferred into 24 -well plate and incubated for $24 \mathrm{~h}$ before transfection. Subsequently, $0.05 \mu \mathrm{g}$ of the luciferase reporter construct were cotransfected with $0.2 \mu \mathrm{g} \beta$-galactosidase vector (Ambion), and 5 pmol miR-668 using Lipofectamine $^{\mathrm{TM}} 2000$ (Invitrogen). Cells were collected 48 hours after transfection and 
Kovacs-Nagy et al. WFS1 and aggression

extracted by three consecutive freeze-thaw cycles followed by a centrifugation. Supernatants were used for luciferase assay. Luminescence for luciferase as well as optical density for $\beta$ galactosidase were measured by Varioskan Flash Multimode Reader, luciferase activity data were normalized by $\beta$-galactosidase activities. All experiments were carried out in triplicates. Statistical analysis of normalized reporter data was performed using one-way ANOVA followed by Tukey-Kramer test (GraphPad InStat 3.05).

\section{Statistical Analysis}

Statistical analyses were carried out using SPSS 19.0 for Windows. Allele and genotype frequency distributions were tested by chi-square analyses. Independent-Samples t-test was applied for assessment of gender differences on self-report scales; their correlation with age was also calculated. Genetic associations were tested by one way analyses of covariance (ANCOVA) assuming a bi-allelic inheritance model and the recessivity of the minor allele. Sex and age were used as covariates, since both were possible confounds. Significant $(p<0.001)$ gender differences were found in aggression and anxiety (males exposed higher aggression and lower anxiety scores). Impulsiveness and depression scores showed no significant sex differences. A significant weak negative correlation was found between age and aggression $\mathrm{r}=-0.073(\mathrm{p}=0.04)$. Other measured scales did not correlate with age. In association analyses nominal level of significance was corrected to $p<0.0007$ based on the Bonferroni correction for multiple testing: $\mathrm{p}=0.05$ was divided by 68 , the number of analyses performed (4 phenotypes $\times 17$ SNPs). Lewontin's $D^{\prime}$ as well as $R^{2}$ values of linkage disequilibrium were determined using HaploView 4.2. [Barrett and others 2005]. 
Kovacs-Nagy et al. WFS1 and aggression

\section{RESULTS}

\section{Higher aggression in AA homozygotes of WFS1 rs1046322}

Table 1 summarizes the most important information for the 17 studied WFS1 SNPs. The average distance between the selected SNPs was $1764 \mathrm{bp}$. Most of them are intronic, but three SNPs in the longest $\left(8^{\text {th }}\right)$ exon and three SNPs in the 3' UTR region were also investigated. The minor allele frequency (MAF) was calculated from the genotype distribution of each polymorphism (see Supplementary Table 1). As our aim was to study common allelic variants, we selected SNPs with MAF $\geq 0.05$.

Figure 1 shows the pairwise linkage disequilibrium (LD) estimates between the studied SNPs of the WFS1 gene region. $R^{2}$ (Figure 1A) as well as $D^{\prime}$ values (Figure 1B) were assessed, the most apparent differences between the two measures could be observed regarding rs 28716718 and rs 1801208 polymorphisms. $R^{2}$ values suggested practically no LD between these two SNPs and the other sites, whereas $D$ ' showed a high level of LD in this region. This finding can be demonstrated in that special form of LD, which can be characterized by the lack of exactly one haplotype, i.e. one allele is freely combined with both alleles of the adjacent site, however the other allele is always present together with one of the two variants of the neighboring polymorphism. The LD structure of WFS1 gene could be characterized by two ( $D^{\prime}$, block definition according to confidence intervals) or three $\left(R^{2}\right.$, block definition according to Four Gamete Rule) haploblocks as labeled in Figure 1. All SNPs were used for the subsequent association analysis.

Individual scores for aggression, impulsiveness, anxiety, and depression were derived from self-report questionnaire data. Mean values are listed for all three genotypes in Table 2. Based on the applied recessive model of the minor allele we compared mean values of minor homozygotes $(\mathrm{mm})$ to those carrying the major allele (MM and $\mathrm{Mm}$ ) for each polymorphism. 
Kovacs-Nagy et al. WFS1 and aggression

Results of these one-way ANCOVAs are depicted in Table 2, sex and age were used as covariates.

The WFS1 rs1046322 showed significant association with Aggression $[\mathrm{F}(1,797)=12.241$, $\mathrm{p}=0.0005, \eta^{2}=0.015$, power $\left.=0.938\right]$, Impulsiveness $\left[\mathrm{F}(1,791)=6.362, \mathrm{p}=0.012, \eta^{2}=0.008\right.$, power $=0.712]$, Anxiety $\left[\mathrm{F}(1,797)=7.801, \mathrm{p}=0.005, \eta^{2}=0.010\right.$, power $\left.=0.797\right]$ and Depression $\left[\mathrm{F}(1,797)=5.074, \mathrm{p}=0.025, \eta^{2}=0.006\right.$, power $\left.=0.614\right]$. These scales are highly related, correlation values reported below are all positive with a $\mathrm{p}$ value lower than 0.0001 Aggression showed a strong correlation with Impulsiveness $(\mathrm{r}=0.43)$ and Anxiety $(\mathrm{r}=0.41)$ and a somewhat weaker correlation with Depression $(r=0.37)$. Impulsiveness was related to both Anxiety $(r=0.27)$ and Depression $(r=0.25)$. Correlation was the highest between Anxiety and Depression $(\mathrm{r}=0.57)$. Participants with the homozygous form of the rs1046322 minor allele $(\mathrm{mm})$ reported significantly higher aggression, they were also more impulsive, more anxious and more depressed than those with genotypes including the major allele (see total scores for each self-report scale in Table 2).

Other WFS1 SNPs did not show significant association with Aggression, Impulsiveness or Anxiety scores. Depression was associated with two other WFS1 SNPs from the $1^{\text {st }}$ haploblock: rs10002743 $\left[\mathrm{F}(1,678)=6.777, \mathrm{p}=0.009, \eta^{2}=0.01\right.$, power $\left.=0.739\right]$ and $\mathrm{rs} 6824720$ $\left[\mathrm{F}(1,673)=6.789, \mathrm{p}=0.009, \eta^{2}=0.01\right.$, power $\left.=0.740\right]$. These SNPs are in strong linkage disequilibrium with each other, but not with the WFS1 rs1046322 polymorphism (see Figure 1).

Due to performing multiple comparisons, the accepted level of significance was corrected to rule out false positive results. From the nominally significant associations (labeled by bold in Table 2) only effect of the WFS1 rs1046322 on Aggression remained significant after the stringent Bonferroni correction (corrected $\mathrm{p}$ value was 0.0007). As highlighted in Table 2, participants with the AA genotype (homozygous for the minor allele) showed the highest 
Kovacs-Nagy et al. WFS1 and aggression

aggression (mean total score $=85.8$ ), whereas subjects with the AG and GG genotypes scored notably lower (their mean total score was 66.3 and 66.4, respectively). It is important to note that only 9 participants out of 801 carried this rare variant (see Supplementary Table 1 for genotype distribution). To test consistency of this genetic effect we calculated mean aggression raw scores for each factor, and compared the average Verbal Aggression, Anger, Hostility and Physical Aggression values for the WFS1 rs1046322 genotypes. Figure 2 shows that aggression level of those with the AA variant are notably higher in all four dimensions as compared to aggression level of carriers of the major allele (AG and GG genotypes). This result indicates that the applied genetic model fits data from the different dimensions of aggression as well as the main construct.

\section{Molecular-functional study of rs1046322}

The rs1046322 SNP which had a significant effect on aggression, impulsiveness, and depression is located in the 3' UTR of the WFS1 gene (see Table 1) and does not fall into any of the identified haploblocks (see Figure 1). This SNP was selected as a putative microRNA binding site polymorphism (miRSNP). According to the PolymiRTS database, the minor allele (A) generates a mismatch in the target site of the seed region of miR-668. To explore whether the rs1046322 polymorphism alters the miR-668 binding efficiency under experimental conditions, a firefly luciferase reporter vector containing the 3' UTR region of the WFS1 gene (pMIR-G) was constructed, and its minor allelic variant was generated by site directed mutagenesis (pMIR-A). The control construct (pMIR-c) possessed an independent sequence with the same length, lacking any complementary region with miR-668. HEK293 cells were cotransfected with miR-668 and with pMIR-G, pMIR-A or pMIR-c, respectively, transfection efficiency was determined by $\beta$-galactosidase reporter vector. The average values of the relative luciferase activities are shown in Figure 3. Expression level was decreased by 
Kovacs-Nagy et al. WFS1 and aggression

miR-668 in the presence of both allelic variants (pMIR-A and pMIR-G) compared to the control (pMIR-c). The wild type 3' UTR variant (pMIR-G) inhibited the luciferase expression upto $28 \%$ of the control, while pMIR-A resulted only in a $47 \%$ silencing effect. ANOVA followed by post hoc analysis showed a statistically significant $(\mathrm{p}<0.05)$ difference between the luciferase reporter activity of the pMIR-G and pMIR-A constructs (ANOVA $p=0.0005$ ). Co-transfection of a control miRNA lacking a binding site in the WFS1 3' UTR did not result in the decrease of relative luciferase activity.

\section{DISCUSSION}

Loss of function mutation of WFS1 gene leads to serious neurodegeneration and early death, suggesting a central role of this protein in the function of the nervous system. Studies on the common WFS1 polymorphisms to date focused primarily on major depression, bipolar depression and suicidality. Kawamoto and colleagues [Kawamoto and others 2004] examined association of mood disorders and certain haplotypes of WFS1. The p.H611R (rs734312) missense exon polymorphism of the WFS1 gene has been linked to mood disorders [Koido and others 2005] in different populations [Zalsman and others 2009]. Suicidal behavior is often a symptom of an underlying psychological disorder, especially mood disorders. Sequeira and colleagues [Sequeira and others 2003] found higher frequency of the WFS1 $611 \mathrm{R} / 611 \mathrm{R}$ genotype in a small sample of completed suicide victims in the general population. This result, however, has not been confirmed by Zalsman and coworkers [Zalsman and others 2009]. A recent study of $4 p$ chromosomal region identified various risk factors of completed suicide in males in several genes including the p.H611R (rs734312) of WFS1, but these results were not significant after the stringent corrections for multiple testing. Interestingly, the risk allele was A in this recent study in opposite to the results of 
Kovacs-Nagy et al. WFS1 and aggression

Sequeira et al. [Sequeira and others 2003] where the G allele emerged as the risk factor of completed suicide.

Based on the above only a handful of association studies investigated the effect of certain WFS1 gene variants on mood disorders and suicide using clinical samples. Results are rather contradictory, which may be a consequence of the small sample size. It is also possible that the most widely investigated WFS1 p.H611R (rs734312) polymorphism is only a marker, being in linkage disequilibrium with another functional polymorphism of the WFS1 gene, such as the microRNA binding site polymorphism (miRSNP) studied here, which has not been assessed previously.

Recent investigations suggested that miRNAs are important regulators of protein synthesis [Bartel 2004]. There is a short (6-8 basepairs) complementary region between the miRNA binding site of the target mRNA and the "seed sequence" of miRNA. A SNP, which influences the microRNA binding (miRSNP), probably has a significant effect on the rate of target protein synthesis. Therefore, miRSNPs represent a novel class of functional polymorphisms, playing an important role in the genetics and pharmacogenetics of complex diseases [Bertino and others 2007]. For example, the miRSNP of dihydrofolate reductase gene was associated with resistance to methotrexate, an anticancer drug [Mishra and others 2007]. There are also a few studies emphasizing the role of the miRSNPs in neuropsychiatric disorders, such as Parkinson's disease and Tourette syndrome [Sethupathy and Collins 2008]. The rs686 of the DRD1gene, previously associated with nicotine dependence, was shown to function as miRSNP [Huang and Li 2009]. Recently, it was demonstrated [Jensen and others 2009] that the rs13212041 in the serotonin receptor 1B gene, which was associated to aggression among college students, disrupts a sequence critical for a miR-96 binding. An additional evidence for this miRSNP was also provided by the same group showing an 
Kovacs-Nagy et al. WFS1 and aggression

association of a haplotype involving rs13212041 with self-reported anger and hostility in young men [Conner and others 2010].

Searching in various databases we found two putative miRSNPs (rs9457, rs1046322) in the WFS1 gene. According to our best knowledge, these common variants have not been tested previously in psychogenetic or diabetes mellitus related association studies. We found that one of these putative miRSNPs, the rs1046322 was associated with aggression and related dimensions. Our in silico studies indicated that the A allele weakens the binding of miR-668. We also provided experimental evidence about the effect of this SNP on miR-668 binding: coexpressing the cloned WFS1 3' UTR possessing A or G alleles with miR-668 resulted in changes of luciferase reporter activity. These results suggest that the rs1046322 SNP has an important molecular function in the regulation of WFS1 gene expression and should be included into further association studies of the WFS1 gene. We hypothesize that the negative regulation of wolframin protein synthesis is less pronounced in the presence of the minor allele of the rs1046322 leading to an overexpression of wolframin and an imbalance in ER stress regulation. Limitation of our hypotheses is the lack of in vitro or in vivo studies that would support the role of miR-668 in the regulation of the wolframin protein synthesis.

Studies to date did not investigate association of WFS1 polymorphisms with impulsivity, aggression or mood characteristics within the non-clinical spectrum. According to the psychiatric literature, these dimensions are interrelated [Apter and others 1990]. Results of the correlation analysis from our present study also showed that Aggression was in a strong correlation with Impulsiveness $(\mathrm{r}=0.43, \mathrm{p}<0.0001)$ ) and Anxiety $(\mathrm{r}=0.41, \mathrm{p}<0.0001)$, although they were assessed with different tools and in populations of different type and size.

The aim of the present study was to detect possible genetic effects of a comprehensive set of WFS1 polymorphisms, involving putative miRSNPs, on psychological dimensions using selfreport data from 801 non-related, healthy Caucasians. Our set of 17 WFS1 SNPs included the 
Kovacs-Nagy et al. WFS1 and aggression

p.H611R (rs734312) polymorphism that was previously associated with mood disorders, however, we did not find any association with impulsivity, aggression or mood characteristics in our large, non-clinical sample. Results from our association analyses revealed a novel risk factor of the impulsive-aggressive tendencies within the non-clinical range: The WFS1 rs1046322 polymorphism, which was identified here as a miRSNP, showed significant association with all the measured scales: Aggression $(p=0.0005)$, Impulsiveness $(p=0.0119)$, Anxiety $(p=0.0053)$ and Depression $(p=0.0246)$. Subjects homozygous for the minor allele (AA) showed higher aggression, higher impulsivity, higher anxiety and higher depression as compared to those without the rare risk genotype. It is important to note, that when correcting the level of significance to rule out false positive results of multiple comparisons only the effect of rs1046322 on Aggression remained significant. Although application of the stringent Bonferroni correction is an important principle in current association studies, we would like to emphasize that most of our nominally significant associations emerged between the WFS1 rs1046322 and the other three dimensions (Impulsiveness, Anxiety and Depression) interrelated with Aggression suggesting a significant and complex effect of this miRSNP on the behavior.

Dimensions of aggression, impulsivity, anxiety, and depression are correlated and are linked to certain psychopathologies, but also cause disturbances in the emotions, decision making and self-regulation within the non-clinical spectrum. Association studies highlight the role of various genetic and environmental predisposing factors in the polygenic model of these behaviors and acknowledge the importance of understanding interaction effects between these components through the study of psychiatric patients as well as the non-clinical population [Courtet and others 2011]. To our knowledge, this is the first association study of a validated microRNA binding site polymorphism in the WFS1 gene. This functional polymorphism was shown as a genetic factor of individual variations in aggression. 
Kovacs-Nagy et al. WFS1 and aggression

Limitation of the present study is the applied self-report method for portraying such a complex dimension as aggression, however, this tool was sufficient for large-scale assessment. We tested 801 subjects, but there were only 9 subjects ( 5 males and 4 females) homozygous with the risk allele (AA) of the microRNA binding site polymorphism (rs1046322) of the WFS1 gene, although the call rate for this SNP was $100 \%$ as a result of double genotyping (see: Supplementary Table 1). Recessivity of the risk allele might be explained by the identified molecular effect of the rs1046322 minor A allele. If microRNA serves as a fine tuning of gene expression, the ER balance could be disturbed only in the presence of both risk alleles. These subjects showed substantially higher levels of aggression (mean value was $86( \pm 22)$ as compared to the overall aggression average: $67( \pm 16)$. Due to the small number of subjects with the risk genotype replication of these findings would be important in an independent non-clinical population. Investigation of this novel functional polymorphism in clinical samples with mood disorders and suicide would also be essential.

\section{ACKNOWLEDGEMENTS}

This work was supported by the Hungarian Scientific Research Funds: OTKA K81466 by Maria Sasvari-Szekely, and OTKA K100845 by Anna Szekely and connected to the scientific program of the NIH R03 TW007656 Fogarty International Research grant awarded to Maria Sasvari-Szekely. This project was also supported by the János Bolyai Research Scholarship (BO/00089/10/5) of the Hungarian Academy of Sciences. Corresponding author has reviewed publication policy and authors have no conflict of interest to declare. 
Kovacs-Nagy et al. WFS1 and aggression

\section{LEGENDS TO THE FIGURES}

Figure 1. Linkage disequilibrium plots of the WFS1 SNPs.

A: $\mathrm{R}^{2}(\%)$ measure of linkage disequilibrium between any pair of SNPs, as determined by HaploView, version 4.2. The gray-scale spectrum indicates pairwise $\mathrm{R}^{2}$ values ranging from black $\left(\mathrm{R}^{2}=1\right)$ to white $\left(\mathrm{R}^{2}=0\right)$. B: Pairwise LD values expressed by D' (HaploView v4.2). Red squares indicate $100 \%$ LD.

Figure 2. The effect of the rs1046322 WFS1 SNP in the Buss-Perry Aggression Scale. Mean raw score values with standard errors of mean for the 4 aggression factors are shown.

Figure 3. The silencing effect of the rs1046322

HEK293 cells were cotransfected with miR-668 and with pMIR-G, pMIR-A or pMIR-c construct, respectively. Relative luciferase activities (mean \pm SD) from three independent experiments are shown. ANOVA showed a statistically significant difference between the luciferase activity of pMIR-A and pMIR-G constructs $(p=0.0005)$.

John Wiley \& Sons, Inc. 
Kovacs-Nagy et al. WFS1 and aggression

\section{REFERENCES}

Apter A, van Praag HM, Plutchik R, Sevy S, Korn M, Brown SL. 1990. Interrelationships among anxiety, aggression, impulsivity, and mood: a serotonergically linked cluster? Psychiatry Res 32(2):191-199.

Barrett JC, Fry B, Maller J, Daly MJ. 2005. Haploview: analysis and visualization of LD and haplotype maps. Bioinformatics 21(2):263-265.

Barrett TG, Bundey SE, Macleod AF. 1995. Neurodegeneration and diabetes: UK nationwide study of Wolfram (DIDMOAD) syndrome. Lancet 346(8988):1458-1463.

Bartel DP. 2004. MicroRNAs: genomics, biogenesis, mechanism, and function. Cell 116(2):281-297.

Bertino JR, Banerjee D, Mishra PJ. 2007. Pharmacogenomics of microRNA: a miRSNP towards individualized therapy. Pharmacogenomics 8(12):1625-1627.

Buss AH, Perry M. 1992. The aggression questionnaire. J Pers Soc Psychol 63(3):452-459.

Conner TS, Jensen KP, Tennen H, Furneaux HM, Kranzler HR, Covault J. 2010. Functional polymorphisms in the serotonin 1B receptor gene (HTR1B) predict self-reported anger and hostility among young men. Am J Med Genet B Neuropsychiatr Genet 153B(1):67-78.

Courtet P, Gottesman, II, Jollant F, Gould TD. 2011. The neuroscience of suicidal behaviors: what can we expect from endophenotype strategies? Transl Psychiatry 1.

Fawcett KA, Wheeler E, Morris AP, Ricketts SL, Hallmans G, Rolandsson O, Daly A, Wasson J, Permutt A, Hattersley AT, Glaser B, Franks PW, McCarthy MI, Wareham NJ, Sandhu MS, Barroso I. 2010. Detailed investigation of the role of common and low-frequency WFS1 variants in type 2 diabetes risk. Diabetes 59(3):741-746.

John Wiley \& Sons, Inc. 
Kovacs-Nagy et al. WFS1 and aggression

Fonseca SG, Fukuma M, Lipson KL, Nguyen LX, Allen JR, Oka Y, Urano F. 2005. WFS1 is a novel component of the unfolded protein response and maintains homeostasis of the endoplasmic reticulum in pancreatic beta-cells. J Biol Chem 280(47):39609-39615.

Fonseca SG, Ishigaki S, Oslowski CM, Lu S, Lipson KL, Ghosh R, Hayashi E, Ishihara H, Oka Y, Permutt MA, Urano F. 2010. Wolfram syndrome 1 gene negatively regulates ER stress signaling in rodent and human cells. J Clin Invest 120(3):744-755.

Huang W, Li MD. 2009. Differential allelic expression of dopamine D1 receptor gene (DRD1) is modulated by microRNA miR-504. Biol Psychiatry 65(8):702-705.

Inoue H, Tanizawa Y, Wasson J, Behn P, Kalidas K, Bernal-Mizrachi E, Mueckler M, Marshall H, Donis-Keller H, Crock P, Rogers D, Mikuni M, Kumashiro H, Higashi K, Sobue G, Oka Y, Permutt MA. 1998. A gene encoding a transmembrane protein is mutated in patients with diabetes mellitus and optic atrophy (Wolfram syndrome). Nat Genet 20(2):143-148.

Ishihara H, Takeda S, Tamura A, Takahashi R, Yamaguchi S, Takei D, Yamada T, Inoue H, Soga H, Katagiri H, Tanizawa Y, Oka Y. 2004. Disruption of the WFS1 gene in mice causes progressive beta-cell loss and impaired stimulus-secretion coupling in insulin secretion. Hum Mol Genet 13(11):1159-1170.

Jensen KP, Covault J, Conner TS, Tennen H, Kranzler HR, Furneaux HM. 2009. A common polymorphism in serotonin receptor $1 \mathrm{~B}$ mRNA moderates regulation by miR-96 and associates with aggressive human behaviors. Mol Psychiatry 14(4):381-389.

Kato T, Ishiwata M, Yamada K, Kasahara T, Kakiuchi C, Iwamoto K, Kawamura K, Ishihara H, Oka Y. 2008. Behavioral and gene expression analyses of Wfs1 knockout mice as a possible animal model of mood disorder. Neurosci Res 61(2):143-158. 
Kovacs-Nagy et al. WFS1 and aggression

Kawamoto T, Horikawa Y, Tanaka T, Kabe N, Takeda J, Mikuni M. 2004. Genetic variations in the WFS1 gene in Japanese with type 2 diabetes and bipolar disorder. Mol Genet Metab 82(3):238-245.

Kesner Y, Zohar J, Merenlender A, Gispan I, Shalit F, Yadid G. 2009. WFS1 gene as a putative biomarker for development of post-traumatic syndrome in an animal model. Mol Psychiatry 14(1):86-94.

Koido K, Koks S, Nikopensius T, Maron E, Altmae S, Heinaste E, Vabrit K, Tammekivi V, Hallast P, Kurg A, Shlik J, Vasar V, Metspalu A, Vasar E. 2005. Polymorphisms in wolframin (WFS1) gene are possibly related to increased risk for mood disorders. Int J Neuropsychopharmacol 8(2):235-244.

Lemonde S, Turecki G, Bakish D, Du L, Hrdina PD, Bown CD, Sequeira A, Kushwaha N, Morris SJ, Basak A, Ou XM, Albert PR. 2003. Impaired repression at a 5hydroxytryptamine $1 \mathrm{~A}$ receptor gene polymorphism associated with major depression and suicide. J Neurosci 23(25):8788-8799.

Luuk H, Plaas M, Raud S, Innos J, Sutt S, Lasner H, Abramov U, Kurrikoff K, Koks S, Vasar E. 2009. Wfs1-deficient mice display impaired behavioural adaptation in stressful environment. Behav Brain Res 198(2):334-345.

M. M, Lesperance. 2010. Human Genetics Laboratory Low-Frequency Hearing Loss WFS1 Gene Mutation and Polymorphism Database. http://www.khri.med.umich.edu/research/lesperance lab/low freq.php.

Mishra PJ, Humeniuk R, Longo-Sorbello GS, Banerjee D, Bertino JR. 2007. A miR-24 microRNA binding-site polymorphism in dihydrofolate reductase gene leads to methotrexate resistance. Proc Natl Acad Sci U S A 104(33):13513-13518.

Must A, Koks S, Vasar E, Tasa G, Lang A, Maron E, Vali M. 2009. Common variations in 4p locus are related to male completed suicide. Neuromolecular Med 11(1):13-19. 
Kovacs-Nagy et al. WFS1 and aggression

Muszbek K, Szekely A, Balogh EM, Molnar M, Rohanszky M, Ruzsa A, Varga K, Szollosi

M, Vadasz P. 2006. Validation of the Hungarian translation of Hospital Anxiety and Depression Scale. Qual Life Res 15(4):761-766.

Oslowski CM, Urano F. 2011. The binary switch that controls the life and death decisions of ER stressed beta cells. Current opinion in cell biology 23(2):207-215.

Patton JH, Stanford MS, Barratt ES. 1995. Factor structure of the Barratt impulsiveness scale. J Clin Psychol 51(6):768-774.

Rigoli L, Lombardo F, Di Bella C. 2011. Wolfram syndrome and WFS1 gene. Clin Genet 79(2):103-117.

Sequeira A, Kim C, Seguin M, Lesage A, Chawky N, Desautels A, Tousignant M, Vanier C, Lipp O, Benkelfat C, Rouleau G, Turecki G. 2003. Wolfram syndrome and suicide: Evidence for a role of WFS1 in suicidal and impulsive behavior. Am J Med Genet B Neuropsychiatr Genet 119B(1):108-113.

Sethupathy P, Collins FS. 2008. MicroRNA target site polymorphisms and human disease. Trends Genet 24(10):489-497.

Swift M, Swift RG. 2000. Psychiatric disorders and mutations at the Wolfram syndrome locus. Biol Psychiatry 47(9):787-793.

Swift RG, Sadler DB, Swift M. 1990. Psychiatric findings in Wolfram syndrome homozygotes. Lancet 336(8716):667-669.

Takei D, Ishihara H, Yamaguchi S, Yamada T, Tamura A, Katagiri H, Maruyama Y, Oka Y. 2006. WFS1 protein modulates the free $\mathrm{Ca}(2+)$ concentration in the endoplasmic reticulum. FEBS Lett 580(24):5635-5640.

Yurimoto S, Hatano N, Tsuchiya M, Kato K, Fujimoto T, Masaki T, Kobayashi R, Tokumitsu H. 2009. Identification and characterization of wolframin, the product of the wolfram 
Kovacs-Nagy et al. WFS1 and aggression

syndrome gene (WFS1), as a novel calmodulin-binding protein. Biochemistry 48(18):3946-3955.

Zalsman G, Mann MJ, Huang YY, Oquendo MA, Brent DA, Burke AK, Ellis SP, Mann JJ. 2009. Wolframin gene H611R polymorphism: no direct association with suicidal behavior but possible link to mood disorders. Prog Neuropsychopharmacol Biol Psychiatry 33(4):707-710.

John Wilez̉ \& Sons, Inc. 


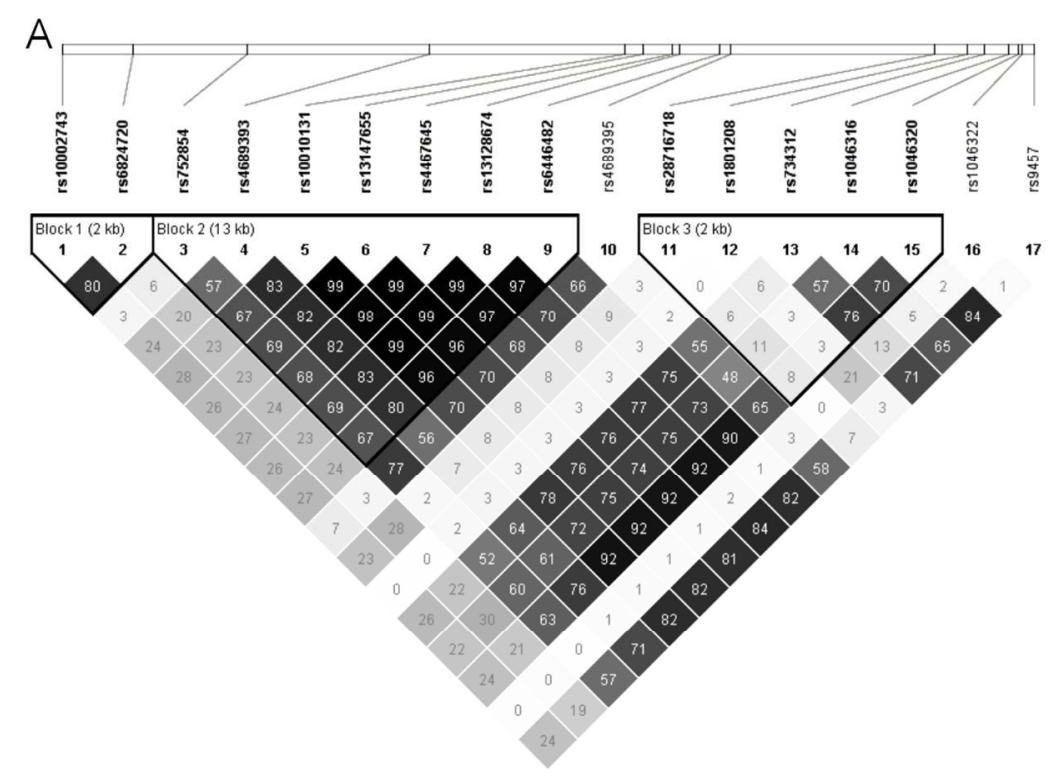

B
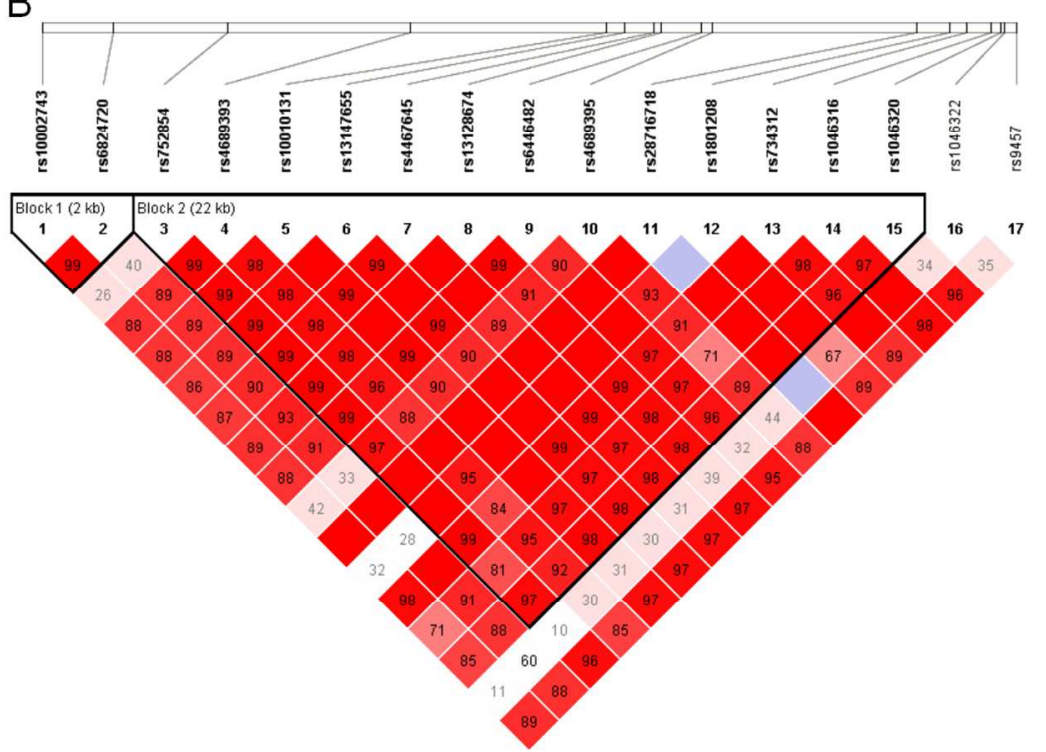

Linkage disequilibrium plots of the WFS1 SNPs.

A: $R^{2}(\%)$ measure of linkage disequilibrium between any pair of SNPs, as determined by HaploView, version 4.2. The gray-scale spectrum indicates pairwise $R 2$ values ranging from black $\left(R^{2}=1\right)$ to white $\left(R^{2}=0\right)$. $B$ : Pairwise LD values expressed by $D^{\prime}$ (HaploView v4.2). Red squares indicate $100 \%$ linkage.

$$
319 \times 475 \mathrm{~mm} \text { (96 x } 96 \text { DPI) }
$$

John Wiley \& Sons, Inc. 


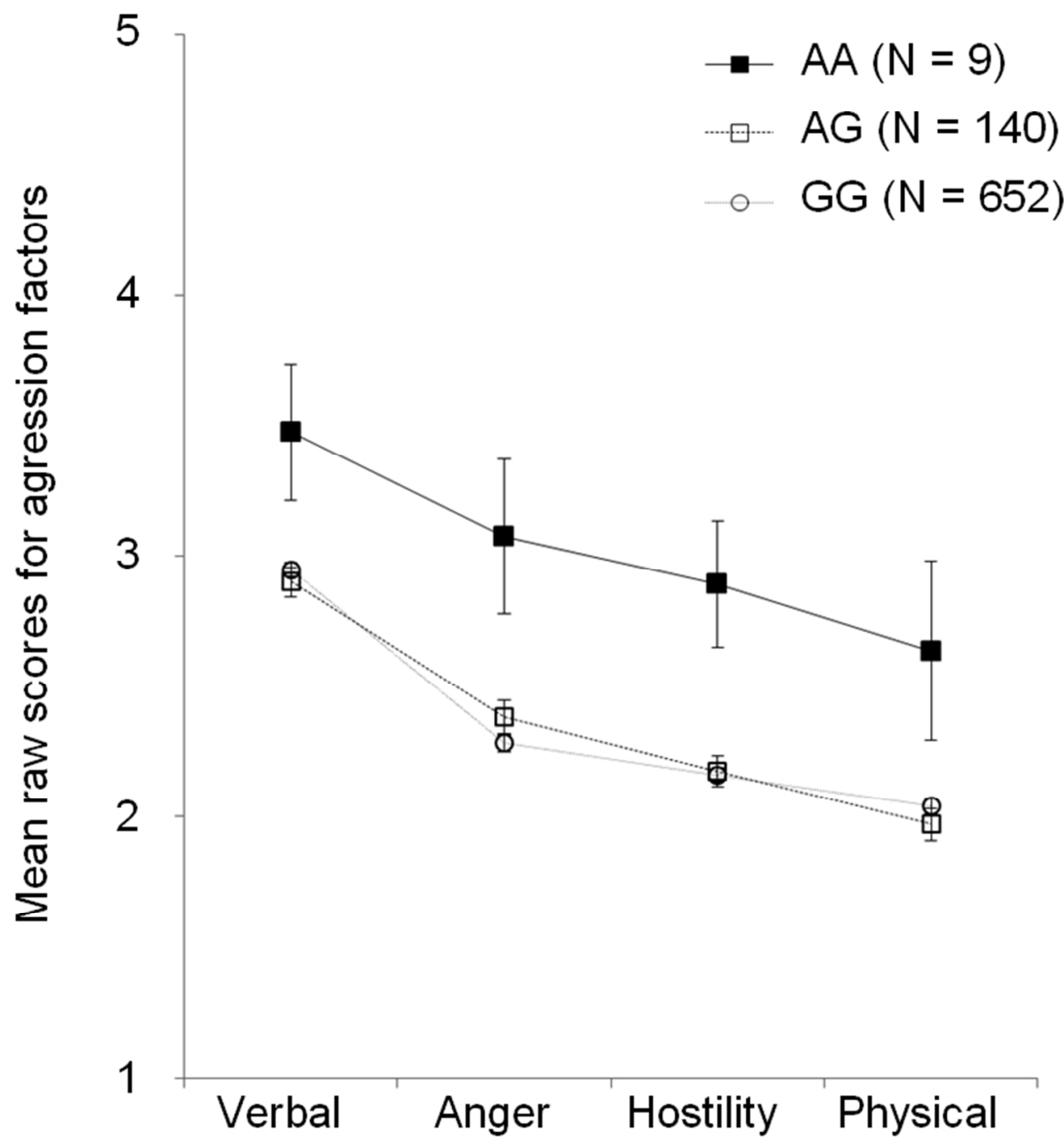

The effect of the rs1046322 WFS1 SNP in the Buss-Perry Aggression Scale. Mean raw score values with standard errors of mean for the 4 agression factors are shown. $210 \times 220 \mathrm{~mm}(96 \times 96 \mathrm{DPI})$ 


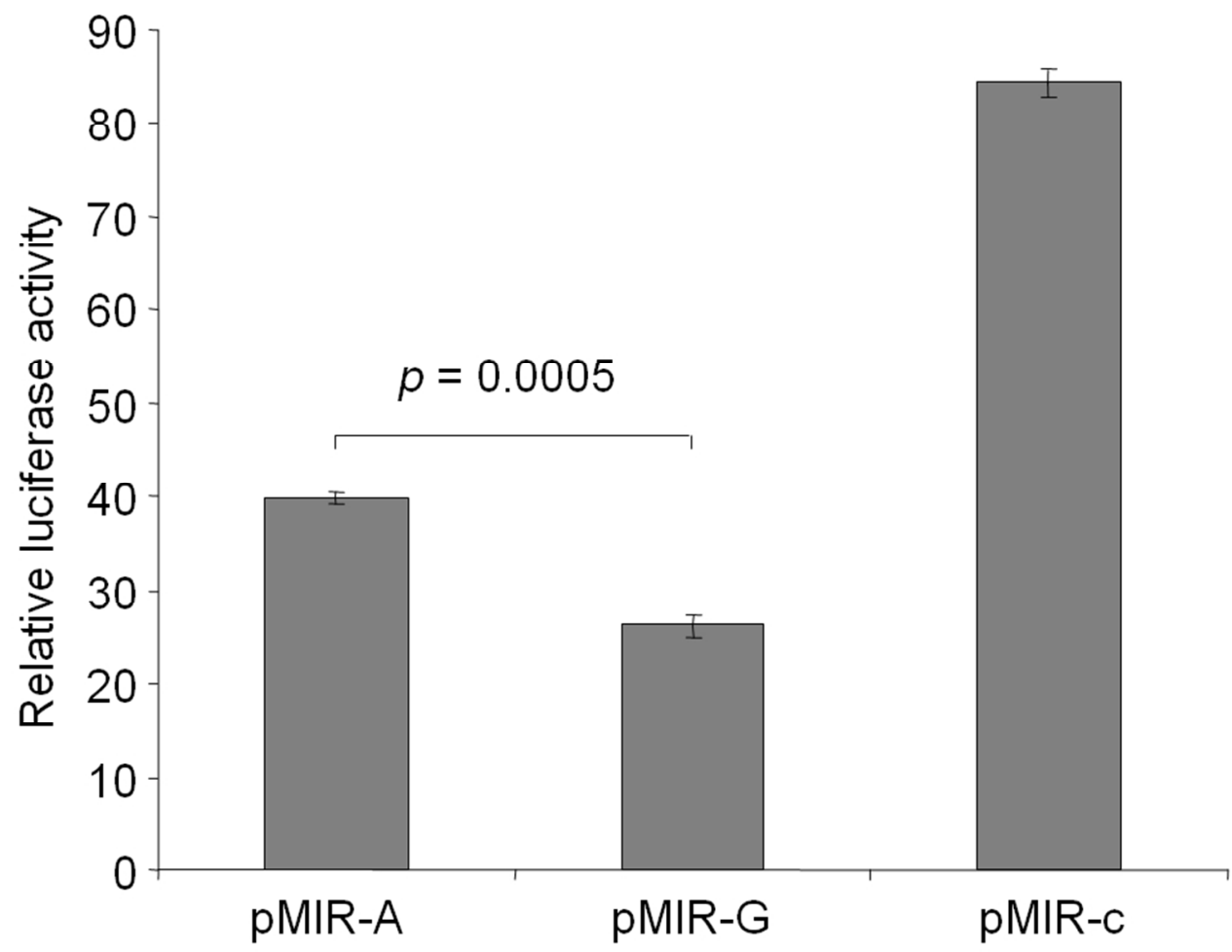

HEK293 cells were cotransfected with miR-668 and with pMIR-G, pMIR-A or pMIR-c construct, respectively. Relative luciferase activities (mean \pm SD) from three independent experiments are shown. ANOVA showed a statistically significant difference between the luciferase activity of pMIR-A and pMIR-G constructs ( $p=$ $0.0005)$.

$260 \times 196 \mathrm{~mm}(96 \times 96 \mathrm{DPI})$ 
2

3

4

5

6

7

8

9

10

11

12

13

14

15

16

17

18

19

20

21

22

23

24

25

26

27

28

29

30

31

32

33

34

35

36

37

38

39

40

41

42

43

44

45

46

47

48

49

50

51

52

53

54

55

56

57

58

59

60

Kovacs-Nagy et al. WFS1 and aggression

Table 1. Main characteristics of the selected WFS1 SNPs.

\begin{tabular}{llccc}
\hline \multicolumn{1}{c}{$\begin{array}{c}\text { dbSNP } \\
\text { number }\end{array}$} & $\begin{array}{c}\text { Genomic } \\
\text { position }\end{array}$ & $\begin{array}{c}\text { Inter-SNP } \\
\text { distance (bp) }\end{array}$ & Type & MAF* \\
\hline rs10002743 & 6276581 & & intron 2 & 0.191 \\
rs6824720 & 6278647 & 2066 & intron 2 & 0.159 \\
rs752854 & 6281961 & 3314 & intron 3 & 0.310 \\
rs4689393 & 6287241 & 5280 & intron 3 & 0.435 \\
rs10010131 & 6292915 & 5674 & intron 4 & 0.391 \\
rs13147655 & 6293474 & 559 & intron 6 & 0.384 \\
rs4467645 & 6294305 & 831 & intron 7 & 0.394 \\
rs13128674 & 6294517 & 212 & intron 7 & 0.409 \\
rs6446482 & 6295693 & 1176 & intron 7 & 0.398 \\
rs4689395 & 6295985 & 292 & intron 7 & 0.359 \\
rs28716718 & 6301910 & 5925 & intron 8 & 0.053 \\
rs1801208 & 6302889 & 979 & exon 8 (p.R456H) & 0.050 \\
rs734312 & 6303354 & 465 & exon 8 (p.H611R) & 0.462 \\
rs1046316 & 6304087 & 733 & exon 8 (p.S855S) & 0.341 \\
rs1046320 & 6304344 & 257 & 3' UTR & 0.403 \\
rs1046322 & 6304448 & 104 & 3' UTR & 0.099 \\
rs9457 & 6304799 & 351 & 3' UTR & 0.433 \\
\hline
\end{tabular}

John Wiley \& Sons, Inc. 
Kovacs-Nagy et al. WFS1 and aggression

Table 2. Association of WFS1 genotypes with self-report measures

Mean values of the total score are presented for each genotype

*significant with Bonferroni correction

\begin{tabular}{|c|c|c|c|c|c|c|c|c|c|c|c|c|c|c|c|c|}
\hline \multirow[b]{2}{*}{ WFS1 SNP } & \multicolumn{4}{|c|}{ AGGRESSION (BUSS-PERRY) } & \multicolumn{4}{|c|}{ IMPULSIVITY (BARRAT) } & \multicolumn{4}{|c|}{ ANXIETY (HADS) } & \multicolumn{4}{|c|}{ DEPRESSION (HADS) } \\
\hline & $(\mathbf{M M})$ & $(\mathbf{M m})$ & $(\mathbf{m m})$ & $\mathbf{p}$ & (MM) & $(\mathbf{M m})$ & $(\mathbf{m m})$ & $\mathbf{p}$ & (MM) & $(\mathbf{M m})$ & $(\mathbf{m m})$ & $\mathbf{p}$ & (MM) & $(\mathbf{M m})$ & $(\mathbf{m m})$ & p \\
\hline rs 10002743 & 66.2 & 68.1 & 66.1 & 0.8711 & 58.3 & 59.8 & 61.1 & 0.2133 & 5.7 & 5.7 & 6.5 & 0.3196 & 2.5 & 2.7 & 3.8 & 0.0094 \\
\hline rs6824720 & 66.4 & 67.1 & 64.1 & 0.5821 & 58.8 & 59.7 & 61.6 & 0.2611 & 5.7 & 5.6 & 6.7 & 0.3235 & 2.5 & 2.7 & 4.1 & 0.0094 \\
\hline rs752854 & 67.5 & 66.5 & 66.8 & 0.8727 & 58.6 & 58.8 & 57.9 & 0.5744 & 5.7 & 5.5 & 5.3 & 0.6456 & 2.7 & 2.6 & 2.6 & 0.9468 \\
\hline rs4689393 & 66.2 & 67.5 & 66.6 & 0.9975 & 58.0 & 59.4 & 59.4 & 0.5180 & 5.5 & 5.7 & 5.6 & 0.9226 & 2.4 & 2.7 & 2.7 & 0.6312 \\
\hline rs10010131 & 66.8 & 66.2 & 64.8 & 0.3542 & 58.1 & 59.8 & 58.5 & 0.6086 & 5.8 & 5.9 & 5.4 & 0.2070 & 2.6 & 2.5 & 2.7 & 0.7347 \\
\hline rs13147655 & 66.6 & 67.4 & 66.1 & 0.7131 & 57.9 & 59.4 & 58.7 & 0.9993 & 5.7 & 5.7 & 5.5 & 0.4438 & 2.6 & 2.7 & 2.8 & 0.7246 \\
\hline rs4467645 & 66.5 & 67.2 & 66.2 & 0.7985 & 58.3 & 59.4 & 58.9 & 0.9656 & 5.6 & 5.8 & 5.6 & 0.6563 & 2.6 & 2.6 & 2.7 & 0.6657 \\
\hline rs13128674 & 66.9 & 67.4 & 67.2 & 0.8759 & 58.1 & 58.8 & 59.0 & 0.6345 & 5.5 & 5.6 & 5.6 & 0.8510 & 2.7 & 2.7 & 2.9 & 0.6256 \\
\hline rs6446482 & 66.3 & 67.3 & 67.0 & 0.8364 & 57.8 & 59.4 & 59.1 & 0.6555 & 5.5 & 5.7 & 5.6 & 0.8530 & 2.5 & 2.6 & 2.8 & 0.4933 \\
\hline rs4689395 & 66.6 & 66.6 & 67.0 & 0.8654 & 58.6 & 59.2 & 59.0 & 0.9261 & 5.7 & 5.7 & 5.5 & 0.7063 & 2.6 & 2.6 & 2.7 & 0.9011 \\
\hline rs28716718 & 66.5 & 66.8 & 72.2 & 0.4960 & 58.8 & 59.9 & 57.0 & 0.6773 & 5.6 & 5.7 & 7.0 & 0.5337 & 2.6 & 2.8 & 3.8 & 0.3753 \\
\hline rs1801208 & 66.6 & 68.5 & 72.5 & 0.5980 & 59.0 & 58.0 & 59.0 & 0.938 & 5.6 & 5.9 & 7.8 & 0.1544 & 2.6 & 2.7 & 2.8 & 0.9045 \\
\hline rs734312 & 66.0 & 67.4 & 66.5 & 0.9215 & 58.7 & 58.8 & 58.9 & 0.8644 & 5.6 & 5.7 & 5.7 & 0.9185 & 2.6 & 2.6 & 2.8 & 0.4407 \\
\hline rs1046316 & 67.3 & 67.5 & 66.4 & 0.8790 & 58.8 & 58.2 & 59.7 & 0.3845 & 5.5 & 5.6 & 5.6 & 0.9628 & 2.7 & 2.7 & 2.8 & 0.8360 \\
\hline rs1046320 & 66.9 & 66.5 & 66.3 & 0.8793 & 58.1 & 59.3 & 58.4 & 0.6323 & 5.7 & 5.8 & 5.3 & 0.1697 & 2.7 & 2.7 & 2.5 & 0.5612 \\
\hline rs1046322 & 66.4 & 66.3 & 85.8 & $0.0005 *$ & 58.6 & 59.1 & 66.9 & 0.0119 & 5.6 & 5.8 & 8.8 & 0.0053 & 2.6 & 2.7 & 4.7 & 0.0246 \\
\hline rs9457 & 66.3 & 67.3 & 65.8 & 0.5462 & 57.8 & 59.2 & 58.2 & 0.6403 & 5.4 & 5.8 & 5.5 & 0.6373 & 2.6 & 2.8 & 2.7 & 0.9249 \\
\hline
\end{tabular}

